\title{
Comparison of ocular higher-order aberrations after SMILE and Wavefront- guided Femtosecond LASIK for myopia
}

\author{
Xiaoqin Chen ${ }^{1}$, Yan Wang ${ }^{1 *}$ (D) Jiamei Zhang ${ }^{1}$, Shun-nan Yang ${ }^{2}$, Xiaojing Li $^{3}$ and Lin Zhang ${ }^{1}$
}

\begin{abstract}
Background: To compare changes in higher-order aberrations (HOAs) following small incision lenticule extraction (SMILE) and wavefront-guided femtosecond laser-assisted in situ keratomileusis (WFG FS-LASIK), and to investigate correlations between preoperative spherical equivalence (SE) and components of HOAs affecting visual quality.

Methods: Sixty-five myopic eyes from 38 patients were enrolled in the study retrospectively, either having undergone SMILE or WFG FS-LASIK. Uncorrected distance visual acuity (UDVA), corrected distance visual acuity (CDVA), refractive error, and individual Zernike coefficients of 3rd- to 6th-order HOAs were measured before and 3 months after the surgeries and were compared using the Mann-Whitney test or Student's t-test. Additional generalized estimating equation analyses (GEE) were used to control for within-subject biases in individual Zernike coefficients between the right and left eyes of the same patients.

Results: There was no significant difference in UDVA or CDVA after WFG FS-LASIK (Mean \pm SD: $-0.02 \pm 0.07$ and $-0.04 \pm 0.22$ respectively, in logMAR) and after SMILE ( $-0.01 \pm 0.06$ and $-0.04 \pm 0.04$ respectively). However, greater vertical coma aberration was found after SMILE $(p=0.036)$. Preoperative SE was correlated to induced horizontal coma $(r=-0.608, p=0.001)$ in WFG FS-LASIK, and correlated to induced vertical coma $(r=-0.459, p=0.003)$ in SMILE.

Conclusions: Both SMILE and WFG FS-LASIK can achieve planned visual outcomes in correcting myopia and myopic astigmatism. However, higher vertical coma was shown in SMILE than WFG FS-LASIK which might be a potentially impact factor for patients' vision under certain lighting conditions and needs further investigation.
\end{abstract}

Keywords: Wavefront aberration, Small incision lenticule extraction, Wavefront-guided femtosecond LASIK, Visual quality, Refractive surgery

\section{Background}

Ocular refractive surgeries require precise corneal correction to achieve ideal visual outcomes and visual quality. Femtosecond laser (FL) utilizes ultrafast pulses to create precise ocular tissue ablation [1], and is commonly used in corneal refractive surgeries to create corneal flaps in femtosecond laser-assisted in situ keratomileusis. Recently, FL has also increasingly been used to create a transparent refractive lenticule in femtosecond lenticule extraction (FLEx) with a lifted corneal flap, and in

\footnotetext{
* Correspondence: wangyan7143@vip.sina.com

${ }^{1}$ Tianjin Eye Hospital \& Eye Institute, Tianjin Key Laboratory of

Ophthalmology and Visual Science, Tianjin Medical University, No 4. Gansu

Rd, Heping District, Tianjin 300020, China

Full list of author information is available at the end of the article
}

small-incision lenticule extraction (SMILE) without a lifted flap [2].

SMILE has been considered as an alternative procedure to conventional laser in-situ keratomileusis (LASIK) because of its potential advantages of reduced denervation, faster resolution of post-operative dry eye, improved biomechanics, and no flap-related risks [3-6]. Previous studies showed that increased higherorder aberrations (HOAs) associated with traditional LASIK could cause glare and halos in night vision. Several recent studies have shown that smaller HOAs were induced after SMILE as compared to traditional LASIK [7, 8]. Wavefront-guided LASIK has been shown to correct preexisting aberrations and to result in less postoperative HOAs [9-11]. FL-assisted ablation in 
wavefront-guided femtosecond LASIK could also potentially reduce induced HOAs [12].

Key procedural differences between SMILE and wavefront-guided femtosecond laser-assisted in situ keratomileusis (WFG FS-LASIK) could contribute to the noted differences in optical qualities following the procedures. SMILE relies on subjective fixation on a target light without eye tracking and iris registration. In contrast, WFG FS-LASIK utilizes iris registration to trace the pupil shift. Pupil shift might affect these two procedures differently, leading to distinct types of HOAs and discordant changes in visual acuity and refraction after SMILE and WFG FS-LASIK.

Therefore, it is necessary to investigate the visual and optical outcomes of WFG FS-LASIK and SMILE and to relate these outcomes to the HOAs induced by the procedures. The present study was the first attempt to determine whether SMILE induces smaller HOAs and achieves better visual outcomes and optical quality than WFG FS-LASIK.

\section{Methods \\ Patients}

This study retrospectively evaluated 65 eyes from 38 patients, including 39 eyes from 23 patients in the SMILE group and 26 eyes from 15 patients in the WFG FSLASIK group. In all of these patients, refractive errors were stable (a change of $\pm 0.50 \mathrm{D}$ or less) for at least 1 year prior to surgery. The corneas of the included eyes were transparent with a central thickness greater than $500 \mu \mathrm{m}$ and a calculated residual stroma not less than $250 \mu \mathrm{m}$. The intraocular pressures (IOPs) of these eyes measured less than $21 \mathrm{mmHg}$. Patients included in this study did not have any of the following systemic or ocular conditions: diabetes mellitus, connective tissue disease, amblyopia, corneal disease, cataracts, glaucoma, or retinal disease. Patients wearing rigid contact lenses were instructed to stop wearing them at least 4 weeks prior to the surgery, and those wearing soft contact lenses were instructed to stop wearing them at least 2 weeks prior.

All patients underwent comprehensive exams before surgery and again 3 months after surgery. The exams included assessments of uncorrected distance visual acuity (UCVA), corrected distance visual acuity (CDVA), manifest and cycloplegic refraction, pupil size, slit-lamp exam, dilated fundus exam, anterior segment tomography (Pentacam-HR, Oculus GmbH, Wetzlar, Germany), IOP with non-contact tonometry (Topcon-CT80; Topcon, Tokyo, Japan), and measurement of wavefront aberrations (Wavescan; VISX, Santa Clara, CA). Full descriptions of the two surgical procedures were provided to all patients, including the potential advantages, disadvantages, and complications. This study was approved by the Ethics Committee of Tianjin Eye Hospital and adhered to the tenets of the Declaration of Helsinki. Informed consent to use any clinical data for analysis and publication was obtained from all patients prior to surgery.

\section{Surgical procedures}

Both the SMILE and the wavefront-guided femtosecond LASIK procedures were performed by an experienced surgeon (Dr. Yan Wang) at the Tianjin Eye Hospital.

\section{SMILE procedure}

A detailed description of the SMILE procedure was previously published and is only briefly summarized here [13]. The SMILE surgery was performed using a 500$\mathrm{kHz}$ Visu Max femtosecond laser (Carl Zeiss, Meditec AG, Jena, Germany) with a laser energy of approximately $170 \mathrm{~nJ}$. Following the application of topical anesthesia (oxybuprocaine eye drops, Benoxil, Santen, Inc., Japan), the patient was required to fixate on an internal target light before corneal suction was initiated. The posterior surface of the lenticule was cut first from the periphery to the center, followed by cutting of the anterior surface from the center to the periphery. The diameter of the refractive lenticule measured from 6 to $6.5 \mathrm{~mm}$ with a transition zone of $0.1 \mathrm{~mm}$. The incision for lenticule retrieval was made at the 12 o'clock position on the cornea and had a length of 2 to $5 \mathrm{~mm}$ with an average of $3.73 \mathrm{~mm}$. The target cap thickness was $110 \mu \mathrm{m}$. A manual spatula was inserted through the small incision to dissect the surface plane, and a pair of forceps was used to extract the intrastromal lenticule.

\section{Wavefront-guided femtosecond LASIK procedure}

WFG FS-LASIK procedures were performed using a VISX STAR S4 excimer laser system (VISX Inc., Santa Clara, USA) after the creation of a $110 \mu \mathrm{m}$ corneal flap with the femtosecond laser. The temporal-hinged corneal flap was made in the requirement of the Ethics Committee of Tianjin Eye Hospital because studies showed horizontal-hinged flap may cause less loss of sensation and presence of dry eye syndrome than superior-hinged flap in LASIK $[14,15]$. Refraction and wavefront information for the treated eyes was collected using a WaveScan system and after being carefully scanned and selected was transferred to the STAR S4 excimer laser system. The operative parameters of the excimer laser were as follows: emission wavelength $193 \mathrm{~nm}$, energy fluence $160 \mathrm{~mJ} / \mathrm{cm}^{2}$, repetition rate $10 \mathrm{~Hz}$, diameter of ablation area 6.0 to $7.0 \mathrm{~mm}$ with transition zone of $0.5 \mathrm{~mm}$. An eye tracker was automatically activated during the laser ablation period. The flap was repositioned then the interface was irrigated with a balanced saline solution. 
After both procedures, $0.3 \%$ ofloxacin (Tarivid, Santen, Inc., Japan) eye drops were instilled 4 times daily for 3 days and $0.1 \%$ fluorometholone (Flumetholon, Santen, Inc., Japan) eye drops were instilled 4 times daily for the first 2 weeks. The $0.1 \%$ flurometholone drops were then gradually tapered, reducing the frequency of instillation every 2 weeks ( 3 times daily, then 2 times daily, and finally once daily). All patients returned for evaluation 3month postoperatively, no complications were found at this examination.

\section{Outcome measurements}

All HOAs were measured by a WaveScan system using a Hartmann-Shack sensor (Wavescan; VISX, Santa Clara, CA) without pharmacological pupil dilatation following 10 min of dark adaptation. The aberrometer was set at a diameter of $5 \mathrm{~mm}$. The absolute coefficients of oblique trefoil, horizontal trefoil, vertical coma, horizontal coma, and spherical aberration were obtained since the magnitudes of the aberration could reflect the optical quality directly [16]. The levels of HOAs were documented as their root mean square values (RMS, in micrometers).

\section{Statistical analyses}

Measured outcomes were analyzed using the SPSS version 20.0 (IBM Inc., New York, USA). The frequency distribution of preoperative and postoperative outcomes were assessed using the Shapiro-Wilks test. Comparisons between preoperative and postoperative data were performed using the Mann-Whitney test for nonnormally distributed data and the Student's $t$-test for normally distributed data. Additional generalized estimating equation (GEE) analyses were conducted to statistically control the contribution of selected (left versus right) eyes of the same patients in preoperative and postoperative individual Zernike coefficients. To examine the effects of preoperative spherical equivalent (SE) on surgical outcomes, correlations between preoperative $\mathrm{SE}$ and the magnitude of individual Zernike coefficients were calculated using the Pearson or Spearman correlation method. All analyses adopted an alpha of 0.05 to determine their statistical significance.

\section{Results}

Patient demographics and preoperative data are summarized in Table 1. There was no significant difference in any of the variables between the SMILE and WFG FSLASIK groups.

\section{Postoperative visual acuity and refraction}

Postoperative outcomes are shown in Table 2. There was no difference in UDVA between the two groups 3 months after surgery. Figure 1 shows no eye with a UDVA worse than 20/32 at the 3-month visit in either group. There was no difference in the percentage of eyes with a change in CDVA between the two groups and no eye lost one or more lines in CDVA (Fig. 2).

Table 2 shows that there was no significant difference in the SE between the two groups 3 months after surgery. Figure 3 shows that the SE was corrected to the targeted level after both types of surgery for all eyes. There was no significant difference $(Z=-0.476$, $p=-0.631)$ in the percentage of eyes achieving

Table 1 Demographics and preoperative data of the study population

\begin{tabular}{llll}
\hline Mean \pm SD (range) & WFG FS-LASIK & SMILE & $P$ value \\
\hline Number of eyes & 26 & 39 & - \\
Age (years) & $24 \pm 5$ & $22 \pm 4$ & $0.186^{\mathrm{b}}$ \\
Gender (\% female) & $40 \%$ & $33 \%$ & 0.542 \\
UDVA (logMAR) & $0.99 \pm 0.37(0.30,1.70)$ & $0.96 \pm 0.28(0.52,1.40)$ & 0.737 \\
CDVA (logMAR) & $0.03 \pm 0.06(0.00,0.22)$ & $0.01 \pm 0.03(0.00,0.10)$ & 0.076 \\
Sphere (D) & $-4.20 \pm 2.65(-10.00,0.00)$ & $-4.41 \pm 1.23(-6.50,-2.00)$ & $0.710^{\mathrm{b}}$ \\
Cylinder (D) & $-2.66 \pm 1.12(-5.50,-1.00)$ & $-2.26 \pm 0.73(-4.00,-1.00)$ & $0.102^{\mathrm{b}}$ \\
SE (D) & $-5.54 \pm 2.40(-10.50,-2.00)$ & $-5.53 \pm 1.24(-8.00,-2.88)$ & $0.998^{\mathrm{b}}$ \\
Ocular aberration (um) & & & 0.249 \\
$Z_{3}^{-3}$ & $0.062 \pm 0.063(0.001,0.211)$ & $0.067 \pm 0.047(0.006,0.206)$ & 0.738 \\
$Z_{3}^{-1}$ & $0.074 \pm 0.045(0.000,0.203)$ & $0.088 \pm 0.068(0.001,0.277)$ & 0.194 \\
$Z_{3}^{1}$ & $0.049 \pm 0.043(0.001,0.168)$ & $0.034 \pm 0.029(0.001,0.126)$ & 0.698 \\
$Z_{3}^{3}$ & $0.040 \pm 0.037(0.000,0.163)$ & $0.043 \pm 0.035(0.000,0.147)$ & $0.280^{\mathrm{b}}$ \\
$Z_{4}^{0}$ & $0.047 \pm 0.027(0.005,0.108)$ & $0.056 \pm 0.034(0.001,0.116)$ & 0.659 \\
$T_{\text {Total HOA }}^{0}$ & $0.158 \pm 0.055(0.078,0.281)$ & $0.166 \pm 0.063(0.057,0.370)$ & \\
\hline
\end{tabular}


Table 2 Visual outcomes and ocular aberrations in the SMILE and wavefront-guided femtosecond LASIK groups 3 months after surgery

\begin{tabular}{llll}
\hline Mean \pm SD (range) & WFG FS-LASIK & SMILE & $P$ value \\
\hline Visual outcomes & & & \\
UDVA (logMAR) & $-0.02 \pm 0.07(-0.18,0.15)$ & $-0.01 \pm 0.06(-0.08,0.15)$ & 0.732 \\
CDVA (logMAR) & $-0.04 \pm 0.06(-0.18,0.15)$ & $-0.04 \pm 0.04(-0.08,0.00)$ & 0.694 \\
SE (D) & $0.01 \pm 0.22(-0.50,0.50)$ & $-0.06 \pm 0.20(-0.63,0.50)$ & 0.128 \\
Ocular Aberration $(\mu \mathrm{m})$ & & & $0.063 \pm 0.045(0.004,0.211)$ \\
$Z_{3}^{-3}$ & $0.072 \pm 0.056(0.001,0.275)$ & $0.163 \pm 0.093(0.026,0.400)$ & 0.342 \\
$Z_{3}^{-1}$ & $0.116 \pm 0.077(0.027,0.304)$ & $0.090 \pm 0.068(0.002,0.250)$ & 0.036 \\
$Z_{3}^{1}$ & $0.084 \pm 0.060(0.001,0.084)$ & $0.048 \pm 0.036(0.004,0.148)$ & 0.399 \\
$Z_{3}^{3}$ & $0.065 \pm 0.055(0.001,0.225)$ & $0.089 \pm 0.048(0.008,0.190)$ & 0.061 \\
$Z_{4}^{0}$ & $0.068 \pm 0.053(0.002,0.225)$ & $0.267 \pm 0.073(0.130,0.439)$ & 0.168 \\
Total HOA & $0.245 \pm 0.094(0.131,0.559)$ & &
\end{tabular}

CDVA =corrected distance visual acuity (spectacles); UDVA =uncorrected distance visual acuity; SE = spherical equivalent; $\mathrm{RMS}=$ root mean square; ${ }^{\text {a }} P$ values were based on Mann-Whitney test for non-normally distributed data unless otherwise specified

emmetropia based on a threshold of $\pm 0.50 \mathrm{D}$ between the two groups (Fig. 4).

\section{Changes in HOAs}

Figure 5 shows that the values of coma, spherical aberration, and total HOA increased significantly in both groups 3 months after surgery as compared to the preoperative data. Table 2 shows significantly higher vertical coma values following SMILE surgery versus wavefrontguided femtosecond LASIK ( $\mathrm{Z}=-2.102, p=0.036$ ); however, no significant difference was found in horizontal coma aberration. The generalized estimating equation (GEE) analysis also revealed a significant difference in vertical coma between the two groups $(\mathrm{W}=4.901$, $p=0.027)$. The trefoil was a little higher following WFG
FS-LASIK in comparison to SMILE, but the difference was not significant.

\section{Correlation between preoperative SE and HOAs}

The left picture in Fig. 6 shows that there was a significant correlation between induced vertical coma and preoperative SE in the SMILE group but not in the WFG FS-LASIK group; there was also a significant difference in the regression slopes between the two groups $(Z=2.123, p=0.038)$. Conversely, the middle one reveals a significant correlation between induced horizontal coma and preoperative SE in the WFG FS-LASIK group but not in the SMILE group; the regression slope, however, was not significantly different between the two groups $(Z=0.044, p=0.965)$.
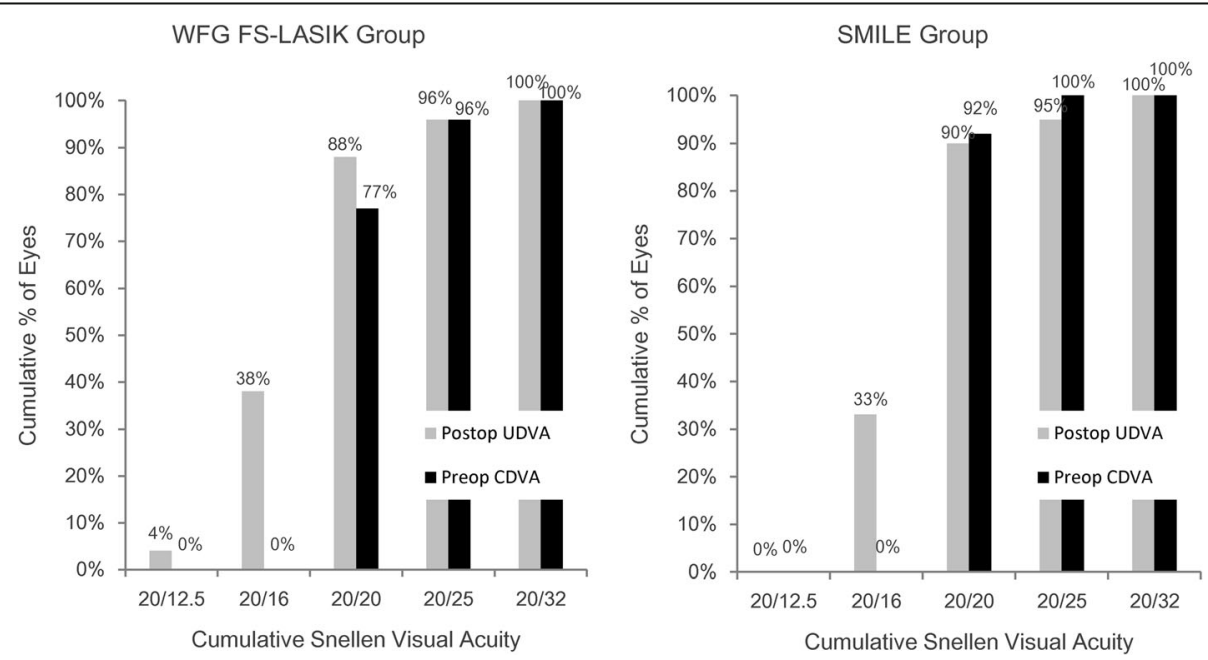

Fig. 1 Cumulative percentages of eyes attaining specified cumulative levels of uncorrected distance visual acuity (UDVA) 3 months after wavefront-guided femtosecond LASIK (WFG FS-LASIK) and small incision lenticule extraction (SMILE) 

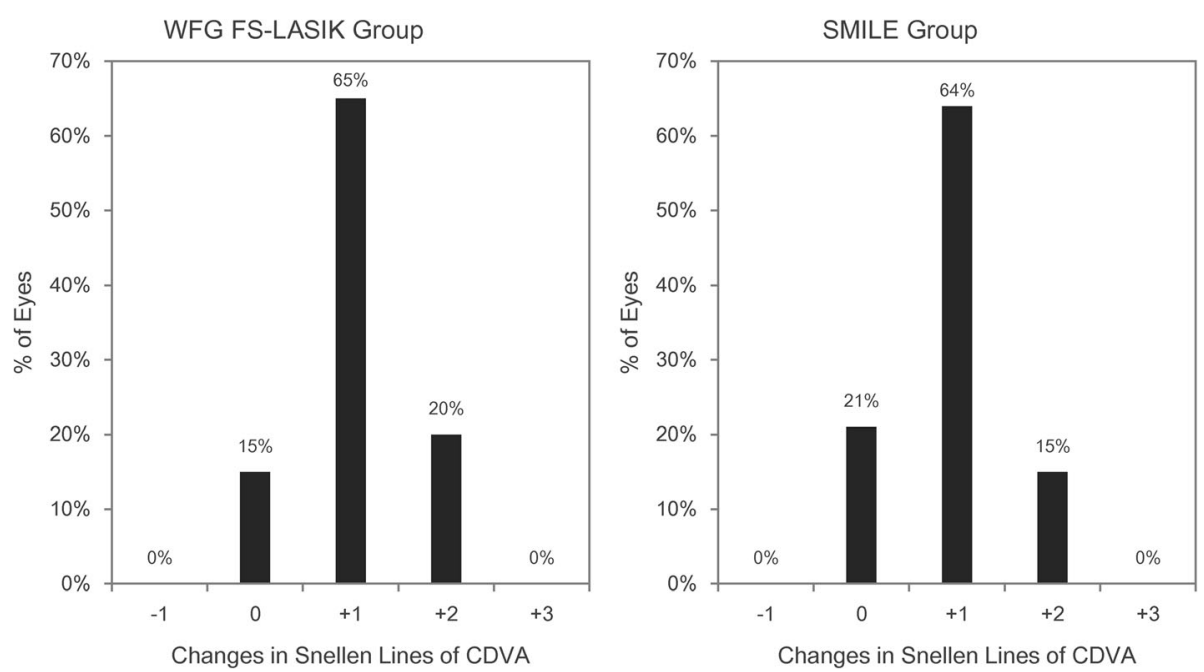

Fig. 2 Distributions of change in Snellen lines for corrected distance visual acuity (CDVA) 3 months after wavefront-guided femtosecond LASIK (WFG FS-LASIK) and small incision lenticule extraction (SMILE)

The right one shows that spherical aberration was significantly correlated to preoperative SE in both groups. There was no difference in the magnitude of the correlation for the two groups as indicated by their respective slopes $(\mathrm{Z}=1.556, p=0.125)$.

\section{Discussion}

The present study investigated post-operative changes in visual outcomes and ocular aberrations in eyes undergoing SMILE and WFG FS-LASIK procedures. Our results show no significant differences in visual outcomes. The surgical efficacy, defined as the percentage of eyes achieving a UDVA of 20/20 or better, was similar for SMILE (90\%) and wavefront-guided femtosecond LASIK (88\%), consistent with previous reports $[7,8,13,17-22]$. The surgical predictability was defined as the percentage of eyes corrected to within $\pm 0.50 \mathrm{D}$ of the intended correction. The present study showed that $97 \%$ of eyes after SMILE and $100 \%$ of eyes after WFG FS-LASIK met this criterion, this is also comparable to earlier studies [17-20, 22-24]. No eye lost one or more lines of CDVA postoperatively in either group. Compared to WFG FSLASIK, the SMILE procedure achieved the same UDVA and CDVA, as well as the targeted refractive correction. Therefore, SMILE can produce safe, efficient, and predictable outcomes similar to the WFG FS-LASIK, the gold standard for correcting HOAs.
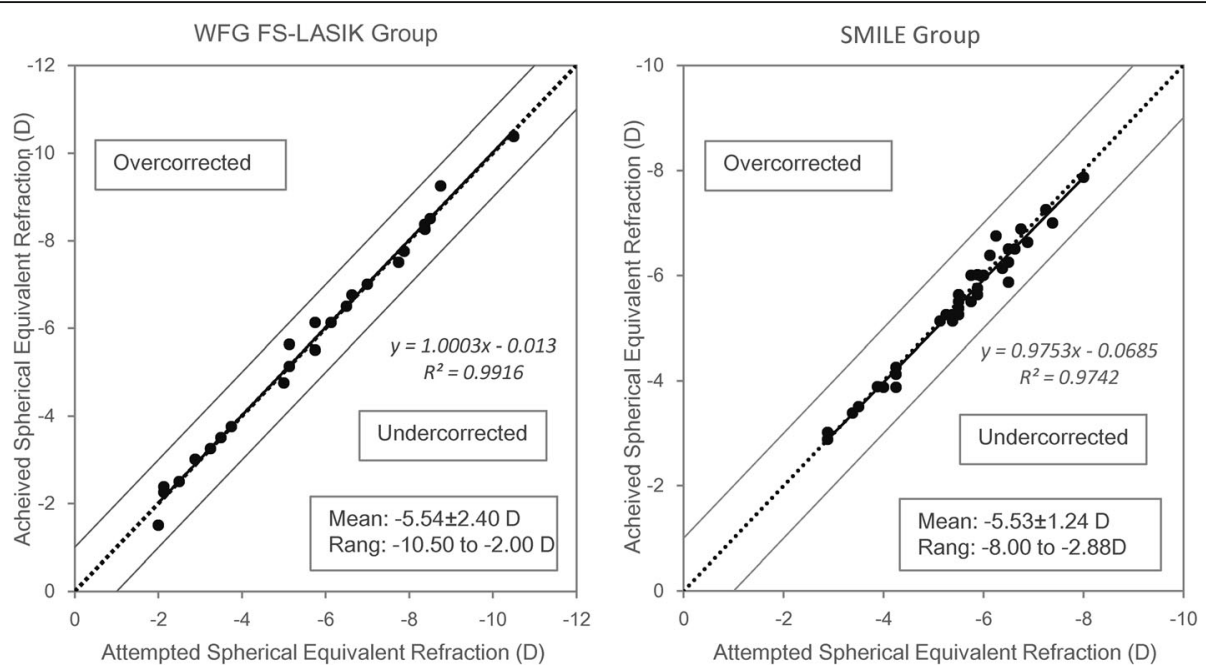

Fig. 3 Relationship between targeted and achieved absolute spherical equivalent (SE) 3 months after surgery. The area above the diagonal dotted line indicates overcorrection and the area below the line indicates undercorrection. Regression lines and corresponding $R^{2}$ was reported for both groups 

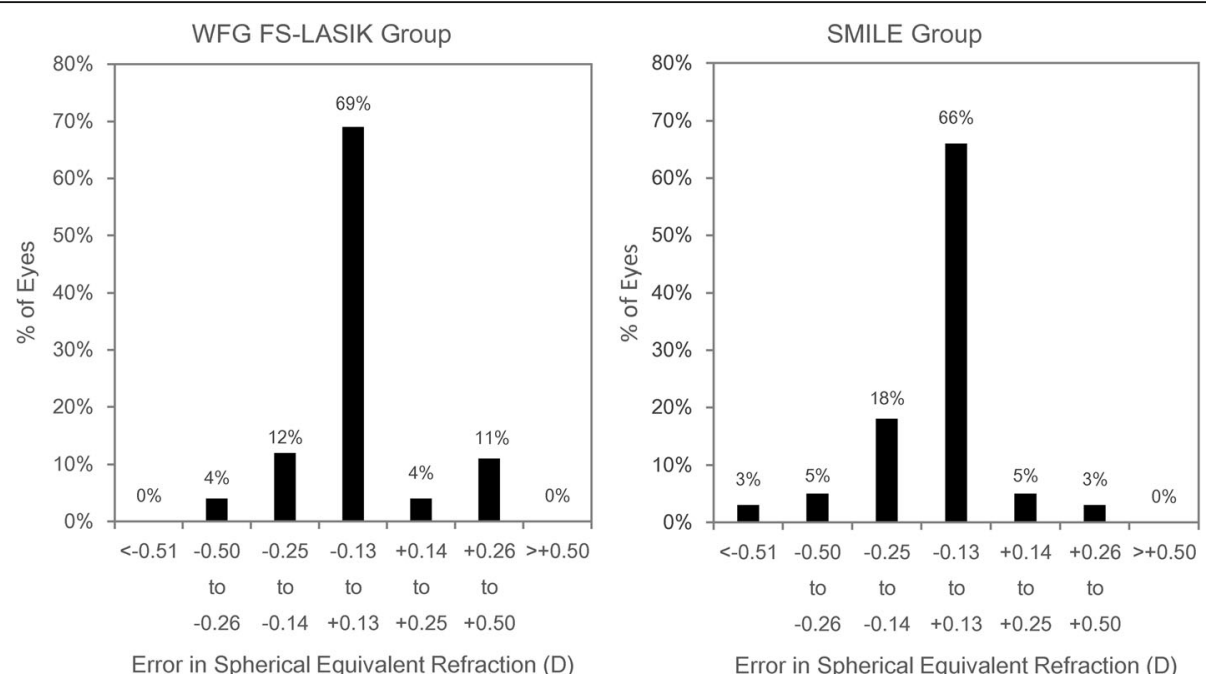

Fig. 4 Percentages of eye of correction error in spherical equivalent (SE) (targeted correction subtracted from achieved correction) 3 months after wavefront-guided femtosecond LASIK (WFG FS-LASIK) and small incision lenticule extraction (SMILE)

There have been several published reports investigating the change in HOAs induced by SMILE $[7,8,12,17$, $25,26]$. The present study showed an increase in total HOAs 3 months after SMILE surgery, which is in line with these earlier findings $[13,17,25,26]$. Consistent with previous reports, the total HOAs in our study also increased after WFG FS-LASIK $[18,21]$. As we know, although the horizontal coma was mirror-symmetric, both negative and positive aberration degrade image quality, thus, the magnitudes of the aberration without regard to the sign still have the same role when evaluating optical performance. [16] Therefore, the absolute Zernike coefficients were analyzed in the present study. Finally, the present study found no significant difference in trefoil, horizontal coma, spherical aberration, and total HOA postoperatively between the two surgeries. A change in vertical coma was the single exception.

A higher vertical coma was found following SMILE as compared to WFG FS-LASIK in the present study. Li and colleagues reported that among the Zernike coefficients, vertical coma showed the greatest increase after SMILE procedure [25]. The authors of that report assumed that the increase in vertical coma after the SMILE procedure was caused by decentration of the lenticule along the vertical axis. In contrast to WFG FSLASIK, the SMILE procedure used in the present study did not have iris registration or eye tracker, which may have resulted in less accurate centration and may explain

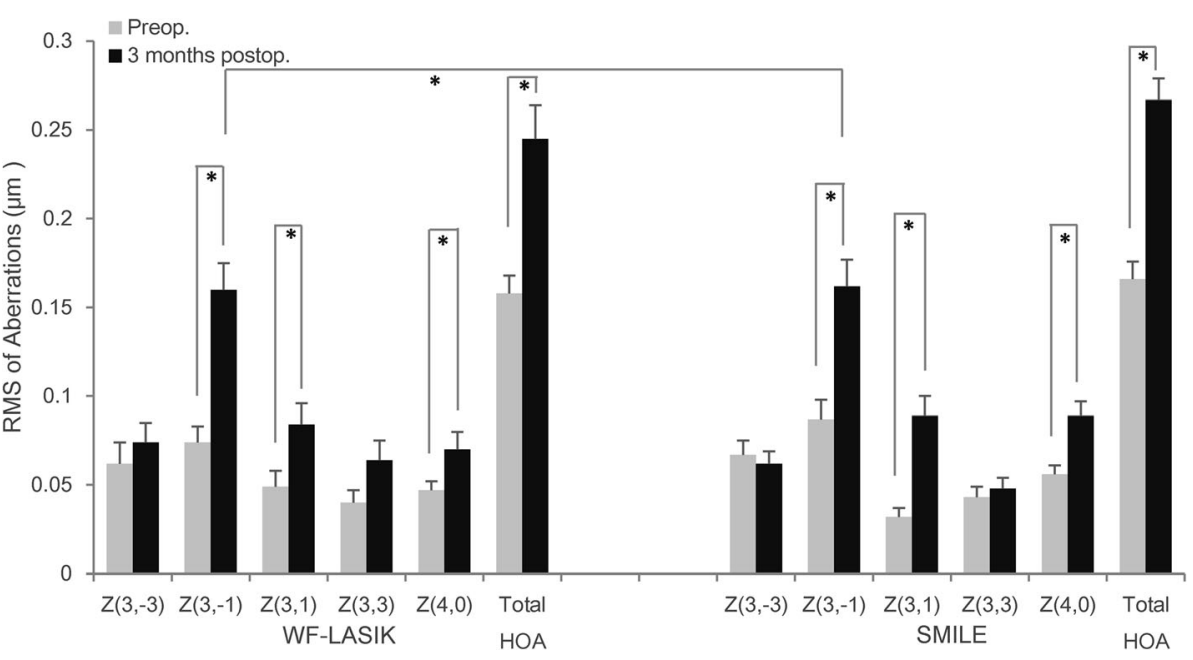

Fig. 5 Root mean square (RMS) of total HOA and individual Zernike coefficients in wavefront-guided femtosecond LASIK (WFG FS-LASIK) and small incision lenticule extraction (SMILE) groups 3 months after surgery. Error bars indicate standard errors and asterisks indicate $p$ values $<0.05$ 

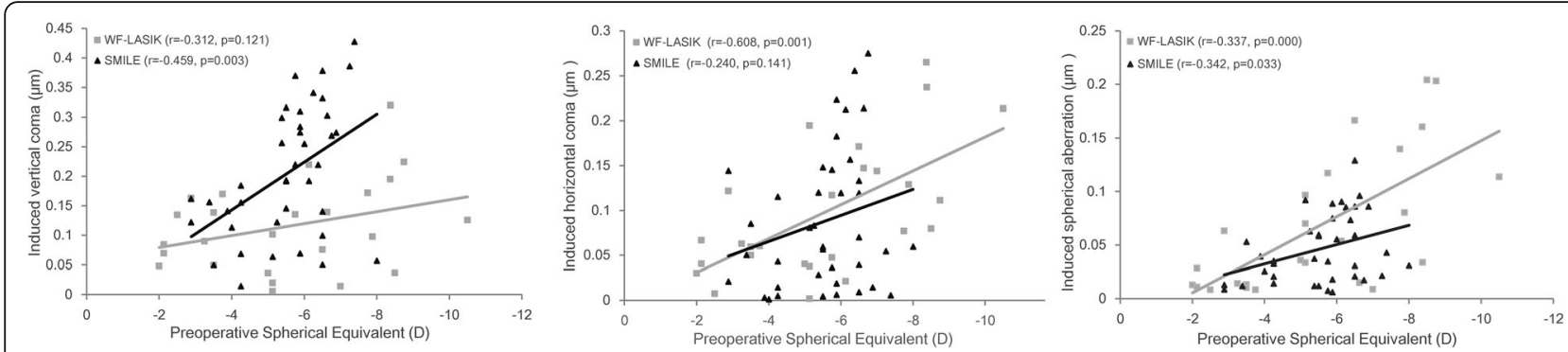

Fig. 6 Scatter plots for preoperative spherical equivalent (SE) and changes in Zernike coefficients measured 3 months after wavefront-guided femtosecond LASIK (WFG FS-LASIK) and small incision lenticule extraction (SMILE). Regression lines and corresponding $r^{2}$ and $p$ values were reported for both groups. Left: SE and vertical coma with SMILE. Middle: SE and horizontal coma with wavefront-guided femtosecond LASIK. Right: Preoperative SE and induced spherical aberration

the higher vertical coma after SMILE surgery. However, ocular aberrations were also influenced by many other factors, such as individual differences in corneal biomechanical properties and corneal wound-healing responses. SMILE is a flapless surgery with a small incision that is made at the superior aspect of the eye. Since superior-hinged flap may cause severe loss of sensation and presence of dry eye syndrome than horizontal-hinged flap in LASIK, the temporal-hinged corneal flap was made in WFG FS-LASIK in the requirement of the Ethics Committee of Tianjin Eye Hospital [14, 15]. Thus, the corneal wound-healing response at the site of the superior incision could induce an asymmetry of aberration in the vertical direction [27]. This hypothesis is also consistent with the nature of HOAs induced by LASIK, with higher horizontal coma likely induced by the flap made on the nasal side of the eye, and vertical coma by the flap at the superior location $[28,29]$.

A novel result was obtained in the present study. There was a significant correlation between the induced vertical coma and preoperative SE in the SMILE group, and a significant correlation between the induced horizontal coma and preoperative SE in the WFG FS-LASIK group. These findings were in line with a previous study which showed that amplitude of induced ocular coma was correlated to the diopter correction and the amount of decentration [30]. Even with the use of eye trackers, decentration may still occur because of several factors related to the patient, surgeon, and machine. Our results suggest that higher refractive corrections increases procedural time, which allows more time for loss of patient fixation and thus induces more aberration [31]. Meanwhile, the wound-healing response also differed due to two main reasons. First, there is a flap created in WFG FS-LASIK, but only a small incision in SMILE, thus SMILE might better maintain the integrity of the cornea. The second reason is the different wound-healing mechanisms related to the power of the correction [32]. It was assumed that more energy would be delivered to the cornea with WFG FS-LASIK because of the higher attempted correction, requiring more tissue to be ablated and increased exposure to the excimer laser. In contrast, energy levels were constant in SMILE and had nothing to do with the attempted correction. Additional studies are required to further evaluate these differences.

The present study also showed a positive change in spherical aberration after both SMILE and WFG FSLASIK. The amount of induced aberration was correlated to the magnitude of the preoperative refractive error. Such an outcome could have been caused by the change in corneal asphericity after refractive surgery. The corneal shape might have changed from its natural prolate aspheric optical architecture to an oblate surface after myopic correction, which would worsen the spherical aberrations [33, 34].

A limitation of the current study is that subjective symptoms were not assessed to further evaluate visual qualities after surgery. In future research, standardized questionnaires could be utilized to find out if the visual quality was significantly impacted by the increased ocular aberration, particularly the vertical coma.

\section{Conclusions}

In conclusion, the present study showed that both SMILE and WFG FS-LASIK were safe, efficient, and predictable procedures for myopic correction, and they produced similar changes in overall ocular aberrations. A higher vertical coma was found in SMILE than WFG FS-LASIK, and this was correlated to preoperative SE. Accurate centration during the SMILE procedure and controlling wound healing might be critical to minimize the induced coma. Further investigation is needed to verify this hypothesis.

\section{Abbreviations}

CDVA: Corrected distance visual acuity; FL: Femtosecond laser; FLEx: Femtosecond lenticule extraction; GEE: Generalized estimating equation analyses; HOAs: Higher-order aberrations; LASIK: Laser in-situ keratomileusisWFG FS-LASIKWavefront-guided femtosecond laser-assisted in situ keratomileusis; SE: Spherical equivalence; SMILE: Small incision lenticule extraction; UDVA: Uncorrected distance visual acuity 


\section{Acknowledgement}

None.

\section{Funding}

This work was supported by a grant from the National Natural Science Foundation of China (No. 81470658)

\section{Availability of data and material}

Available upon request from the first author; Dr. Xiaoqin Chen.

\section{Authors' contributions}

Study concept and design were performed by CXQ and WY. Preparation of the first draft of the manuscript was done by CXQ and revised by YSN and ZJM. Data was analyzed and interpreted by CXQ and YSN. Data collection was performed by ZJM, LXJ and ZL. Review and approval of the manuscrip was performed by WY. All authors read and approved the final manuscript.

\section{Competing interests}

The authors do not have any conflict of interest to report and have no proprietary interest in any of the materials mentioned in this article.

\section{Consent for publication}

Not applicable.

\section{Ethics approval and consent to participate}

This study was approved by the Ethics Committee of Tianjin Eye Hospital and adhered to the tenets of the Declaration of Helsinki. Informed consent to use any clinical data for analysis and publication was obtained from all patients prior to surgery.

\section{Publisher's Note}

Springer Nature remains neutral with regard to jurisdictional claims in published maps and institutional affiliations.

\section{Author details}

${ }^{1}$ Tianjin Eye Hospital \& Eye Institute, Tianjin Key Laboratory of Ophthalmology and Visual Science, Tianjin Medical University, No 4. Gansu Rd, Heping District, Tianjin 300020, China. ${ }^{2}$ Vision Performance Institute, Pacific University College of Optometry, Forest Grove, OR, USA. ${ }^{3}$ First Hospital of Qinhuangdao, Qinhuangdao, Hebei, China.

\section{Received: 13 December 2016 Accepted: 31 March 2017} Published online: 07 April 2017

\section{References}

1. Sugar A. Ultrafast (femtosecond) laser refractive surgery. Curr Opin Ophthalmol. 2002:13:246-9.

2. Kim P, Sutton GL, Rootman DS. Applications of the femtosecond laser in corneal refractive surgery. Curr Opin Ophthalmol. 2011;22:238-44.

3. Moshirfar M, McCaughey MV, Reinstein DZ, Shah R, Santiago-Caban L, Fenzl CR. Small-incision lenticule extraction. J Cataract Refract Surg. 2015;41:652-65.

4. Mohamed-Noriega K, Riau AK, Lwin NC, Chaurasia SS, Tan DT, Mehta JS. Early corneal nerve damage and recovery following small incision lenticule extraction (SMILE) and laser in situ keratomileusis (LASIK). Invest Ophthalmol Vis Sci. 2014;55:1823-34.

5. Xu Y, Yang Y. Dry eye after small incision lenticule extraction and LASIK for myopia. J Refract Surg. 2014;30:186-90.

6. Wu D, Wang Y, Zhang L, Wei S, Tang X. Corneal biomechanical effects: small-incision lenticule extraction versus femtosecond laser-assisted laser in situ keratomileusis. J Cataract Refract Surg. 2014;40:954-62.

7. Ganesh S, Gupta R. Comparison of visual and refractive outcomes following femtosecond laser-assisted LASIK with SMILE in patients with myopia or myopic astigmatism. J Refract Surg. 2014;30:590.

8. Lin F, XU Y, Yang Y. Comparison of the visual results after SMILE and femtosecond laser-assisted LASIK for myopia. J Refract Surg. 2014;30:248.

9. Mello GR, Rocha KM, Santhiago MR, Smadja D, Krueger RR. Applications of wavefront technology. J Cataract Refract Surg. 2012;38:1671-83.

10. Schallhorn SC, Farjo AA, Huang D, et al. Wavefront-guided LASIK for the correction of primary myopia and astigmatism a report by the American Academy of Ophthalmology. Ophthalmology. 2008;115:1249-61.
11. Zhang J, Zhou YH, Li R, Tian L. Visual performance after conventional LASIK and wavefront-guided LASIK with iris-registration: results at 1 year. Int Ophthalmol. 2013;6:498-504.

12. Durrie DS, Kezirian GM. Femtosecond laser versus mechanical keratome flaps in wavefront-guided laser in situ keratomileusis: prospective contralateral eye study. J Cataract Refract Surg. 2005;31:120-6.

13. Shah R, Shah $\mathrm{S}$, Sengupta $\mathrm{S}$. Results of small incision lenticule extraction: all-in-one femtosecond laser refractive surgery. J Cataract Refract Surg. 2011:37:127-37.

14. Donnenfeld ED, Solomon K, Perry HD, et al. The effect of hinge position on corneal sensation and dry eye after LASIK. Ophthalmology. 2003;110:1023-9.

15. Feng YF, Yu JG, Wang DD, et al. The effect of hinge location on corneal sensation and dry eye after LASIK: a systematic review and meta-analysis. Graefes Arch Clin Exp Ophthalmol. 2013;251:357-66.

16. Salmon TO, Van de Pol C. Normal-eye Zernike coefficients and root-meansquare wavefront errors. J Cataract Refract Surg. 2006;32:2064-74.

17. Sekundo W, Gertnere J, Bertelmann T, Solomatin I. One-year refractive results, contrast sensitivity, high-order aberrations and complications after myopic small-incision lenticule extraction (ReLEx SMILE). Graefes Arch Clin Exp Ophthalmol. 2014:252:837-43.

18. Yu CQ, Manche EE. Comparison of 2 femtosecond lasers for flap creation in myopic laser in situ keratomileusis: one-year results. J Cataract Refract Surg. 2015;41:740-8

19. Prakash G, Srivastava D, Suhail M. Femtosecond laser-assisted Wavefrontguided LASIK using a newer generation Aberrometer: 1-year results. J Refract Surg. 2015:31:600-6.

20. Smadja D, Santhiago MR, Tellouck J, et al. Safety and efficacy of wavefrontguided myopic laser in situ keratomileusis using a new wavefront sensor technology: first 100 cases. J Cataract Refract Surg. 2015;41:1588-93.

21. He L, Liu A, Manche EE. Wavefront-guided versus wavefront-optimized laser in situ keratomileusis for patients with myopia: a prospective randomized contralateral eye study. Am J Ophthalmol. 2014;157:1170-8.

22. Liu M, Chen $Y$, Wang $D$, et al. Clinical outcomes after SMILE and Femtosecond laser-assisted LASIK for myopia and myopic astigmatism: a prospective randomized comparative study. Cornea. 2016:35:210-6.

23. Kamiya K, Shimizu K, Igarashi A, Kobashi H. Visual and refractive outcomes of femtosecond lenticule extraction and small-incision lenticule extraction for myopia. Am J Ophthalmol. 2014;157:128-342.

24. Verdaguer $\mathrm{P}$, El-Husseiny MA, Elies $\mathrm{D}$, et al. Small incision lenticule extraction (SMILE) procedure for the correction of myopia and myopic astigmatism. J Emmetropia. 2013:4:191-6.

25. Li M, Zhao J, Miao H, et al. Mild decentration measured by a Scheimpflug camera and its impact on visual quality following SMILE in the early learning curve. Invest Ophthalmol Vis Sci. 2014:55:3886-92.

26. Tan DK, Tay WT, Chan C, Tan DT, Mehta JS. Postoperative ocular higherorder aberrations and contrast sensitivity: femtosecond lenticule extraction versus pseudo small-incision lenticule extraction. J Cataract Refract Surg. 2015;41:623-34.

27. Li X, Wang Y, Dou R. Aberration compensation between anterior and posterior corneal surfaces after small incision lenticule extraction and Femtosecond laser-assisted laser in-situ keratomileusis. Ophthalmic Physiol Opt. 2015;35:540-51.

28. Pallikaris IG, Kymionis GD, Panagopoulou SI, Siganos CS, Theodorakis MA, Pallikaris Al. Induced optical aberrations following formation of a laser in situ keratomileusis flap. J Cataract Refract Surg. 2002:28:1737-41.

29. Potgieter FJ, Roberts C, Cox IG, et al. Prediction of flap response. J Cataract Refract Surg. 2005:31:106-14.

30. Guirao A, Williams DR, Cox IG. Effect of rotation and translation on the expected benefit of an ideal method to correct the eye's higher-order aberrations. Opt Soc Am A Opt Image Sci Vis. 2001;18:1003-15.

31. Padmanabhan P, Mrochen M, Viswanathan D, Basuthkar S. Wavefront aberrations in eyes with decentered ablations. J Cataract Refract Surg. 2009; 35:695-702.

32. Riau AK, Angunawela RI, Chaurasia SS, Lee WS, Tan DT, Mehta JS. Early corneal wound healing and inflammatory responses after refractive lenticule extraction (ReLEx). Invest Ophthalmol Vis Sci. 2011:52:6213-21.

33. Bottos KM, Leite MT, Aventura-Isidro M, et al. Corneal asphericity and spherical aberration after refractive surgery. J Cataract Refract Surg. 2011;37:1109-15.

34. Chen CC, Izadshenas A, Rana MA, Azar DT. Corneal asphericity after hyperopic aser in situ keratomileusis. J Cataract Refract Surg. 2002;28:1539-45. 